\title{
Odd Factor Decomposition of E-Super Magic Graphs
}

P. Hemalatha and V. Nivetha

Department of Mathematics, Vellalar College for Women, Erode - 638 012, Tamilnadu, India; dr.hemalatha@gmail.com, nivethavelumani93@gmail.com

\section{Abstract}

An F-magic labeling in an F-decomposable graph $\mathrm{G}$ of order $\mathrm{p}$ and size $\mathrm{q}$ is a bijection $f: V(G) \cup E(G) \rightarrow\{1,2 \ldots . p+q\}$ such that for every copy $\mathrm{F}$ in the decomposition, $\sum_{v \in V(F)} f(v)+\sum_{e \in E(F)} f(e)$ is constant. The function $\mathrm{f}$ is said to be F-E super magic if $f(E(G))=\{1,2, \cdots q\}$. This article contains, a necessary and some sufficient conditions for some even regular and odd regular graphs $\mathrm{G}$ to have an $(2 k+1)$ - factor E-super magic decomposition, for $k \geq 1$.

Keywords: F-Decomposable Graph, F-E Super Magic Labeling, $(2 k+1)$-Factor E-Super Magic Decomposition of Graphs.

\section{Introduction}

Graph theory is a major tool in computer programming mathematical research, electrical electrical engineering, communication networking and so on.

Labeling of graphs, a special area in graph theory, is useful for broad range of applications in the field of communication, network areas and models for constraint programming over finite domain. Many kinds of labeling have been studied since 1967. A detailed study has been done by Joseph A. Gallian ${ }^{[3]}$. Further, the study on magic labeling was done by J. A. MacDougall[4].

All graphs considered here are finite, simple undirected graphs. Let $G$ be a graph with vertex set $V(G)$ and the edge set $E(G)$ such that the order of $G=|V(G)|=p$ and the size of $G=|E(G)|=q$. A labeling of a graph $G$ is a mapping from a set of vertices (edges) into a set of numbers, usually integers.

A decomposition of $G$ is a partitioning of $G$ into subgraphs $F_{1}, F_{2}, \ldots F_{s}$ such that $E\left(F_{i}\right) \cap E\left(F_{j}\right)=\phi$ for $i \neq j, i, j \in\{1,2, \cdots s\}$ and $\bigcup_{i=1}^{s} E\left(F_{i}\right)=E(G)$ and we write $G=\bigoplus_{i=1}^{s} F_{i}$. If all the sub graphs Fi are isomorphic to a graph $F$ then $G$ is said to be $F$-decomposable. A spanning sub graph of a graph $G$ is called a factor of $G$. If $G$ can be expressed as an edge disjoint sum of factors $H_{1}, H_{2} \cdots H_{k}$ then it is called a factorization of $G$. If each $H_{i} \cong H$, then it is called an $H$-factorization of $G$. If $H$ is $m$ -regular then $G$ is said to have $m$-factor decomposition.

A vertex magic total labeling is a bijection $f$ from $V(G) \cup E(G)$ to the set of integers $\{1,2,3, \ldots p+q\}$ with the property that for every $u \in V(G)$,

$$
f(u)+\sum_{v \in N(u)} f(u v)=k \text { for some constant } k \text {. Such a }
$$


labeling is E-super if $f(E(G))=\{1,2,3, \ldots . q\}$. A graph $\mathrm{G}$ is called E-super vertex magic if it admits an E-super vertex magic labeling. Super vertex magic labeling was introduced by Swaminathan and Jeyanthi ${ }^{[9]}$. E-super vertex magic labeling of graphs has been studied by Marimuthu and Balakrishnan in ${ }^{[5]}$. They proved that, if a graph $\mathrm{G}$ of odd order can be decomposed into two Hamiltonian cycles, then $\mathrm{G}$ is an E-super vertex magic graph. $\mathrm{In}{ }^{[10]}, \mathrm{T}$. $M$ Wang and Guang-Hui gave the generalization of some results stated in ${ }^{[5]}$ using 2 -factor.

A total labeling $f: V(G) \cup E(G) \rightarrow\{1,2 \ldots p+q\}$ is called an F-magic labeling of $\mathrm{G}$ if there exists a positive integer $k$ (called magic constant) such that for every copy F in the decomposition, $\sum_{v \in V(F)} f(v)+\sum_{e \in E(F)} f(e)=k$. A graph $\mathrm{G}$ that admits such a labeling is called an F-magic decomposable graph. An F-magic labeling is called an F-E super magic labeling if $f(E(G))=\{1,2,3, \ldots . q\}$. A graph that admits an F-E super magic labeling is called an F-E super magic decomposable graph. Subbiah and Padimadevi ${ }^{[8]}$ obtained a necessary and sufficient condition for 2-factor decomposition of F-E super magic graphs. In this paper a necessary and some sufficient conditions are obtained for a $(2 k+1)$-factor decomposition of E-super magic graphs.

The results given below are useful to prove some of the theorems.

\subsection{Lemma ${ }^{[5]}$}

If a non-trivial graph $G$ with order $p$ and size $q$ is super vertex magic, then the magic constant $k$ is given by

$$
k=q+\frac{p+1}{2}+\frac{q(q+1)}{p} .
$$

\subsection{Lemma ${ }^{[8]}$}

If a non-trivial $m$-factor decomposable graph $\mathrm{G}$ is $m$-factor E-super decomposable, then the magic constant $k$ is

$$
p q+\frac{p(p+1)}{2}+\frac{q(q+1)}{2 s} \text { where } s \text { is the number of }
$$
$m$-factors of $G$.

\subsection{Lemma $^{[\underline{8}]}$}

If a non-trivial $m$-factor decomposable graph $\mathrm{G}$ is $m$-factor E-super magic decomposable, then the sum of the edge labels, denoted by $k_{e}$, is a constant and it is given by $k_{e}=\frac{q(q+1)}{2 s}$, where $s$ the number of $m$-factors of G.

\subsection{Lemma $^{[8]}$}

Let $\mathrm{G}$ be an $m$-factor decomposable graph and let $g$ be a bijection from $E(G)$ onto $\{1,2,3, \ldots . q\}$. Then $g$ can be extended to an $m$-factor E-super magic labeling of $\mathrm{G}$ if and only if $k_{e}=\sum_{e \in E\left(G^{\prime}\right)} g(e)$ is constant for every $m$-factor $G^{\prime}$ in the decomposition of $\mathrm{G}$.

\subsection{Theorem ${ }^{[6]}$}

Every $m$ and $r$-regular graph has $r$-factor for every positive integer $r$ and $m$, where $r, m \geq 1$.

\section{2. $(2 k+1)$ - Factor Decomposition in E-Super Magic Regular Graphs}

In this section, a necessary and some sufficient conditions are obtained for some even or odd regular graphs $\mathrm{G}$ of even order to have an $(2 k+1)$-factor E-super magic decomposition for $k \geq 1$. It is proved that every $2(2 k+1) r$ - regular graph (i.e., even regular graph) with $2[(2 k+1) r+1]$ order (i.e., even order) and for every $3(2 k+1) r$ regular graph (i.e., odd regular graph) with $3(2 k+1) r+1$ order (i.e., even order) will have an $(2 k+1)$-factor E-super magic decomposition, for $k \geq 1$ and $r=2 k-1$.

Next theorem gives the necessary condition for $m$ factor decomposition of E-super magic graphs.

\subsection{Theorem}

If $\mathrm{G}$ is an $m$-factor decomposable E-super magic graph, then $\mathrm{G}$ is $r$-regular for some integer $r$ that is a multiple of $m$.

\subsubsection{Proof}

Proof follows from Theorem 1.5.

Next theorem shows that the necessary condition is also sufficient for $m=(2 k+1)$, when G is of order $2[(2 k+1) r+1]$ 
and $2(2 k+1) r$-regular, for $k \geq 1$ and $r=2 k-1$.

\subsection{Theorem}

Every graph G with $2[(2 k+1) r+1] \quad$ vertices of $2(2 k+1) r$-regular is $(2 k+1)$-factor E-super magic decomposable, for $k \geq 1$ and $r=2 k-1$.

\subsubsection{Proof}

Let $\mathrm{G}$ be an $2(2 k+1) r$-regular (i.e., even regular graph) of even order say $p=2[(2 k+1) r+1]$. Then by Theorem 1.5, G is decomposed into $(2 k+1)$ - factors say, $G=F_{1}, F_{2}, \cdots F_{s}$ where $F_{i}$ is a $(2 k+1)$ - factor for each $i$, $1 \leq i \leq s$. Since $\mathrm{G}$ is of order $p$ with $s$ number of $(2 k+1)$ factor then the number of edges of G, $q=\frac{(2 k+1) p s}{2}$. Let $l_{i}(e)$ denotes the edge labels corresponding to a factor $F_{i}$, $i=1,2,3, \cdots s$ in the decomposition. Now the labeling of edges in $\mathrm{G}$ is as follows:

\begin{tabular}{|c|c|c|c|}
\hline$l_{1}(\mathrm{e})$ & $l_{2}(\mathrm{e})$ & $\cdots$ & $l_{\mathrm{s}}(\mathrm{e})$ \\
\hline 1 & 2 & $\cdots$ & $\mathrm{S}$ \\
\hline $2 \mathrm{~s}$ & $2 \mathrm{~s}-1$ & $\cdots$ & $\mathrm{s}+1$ \\
\hline $2 \mathrm{~s}+1$ & $4 \mathrm{~s}-1$ & $\ldots$ & $3 \mathrm{~s}$ \\
\hline $4 \mathrm{~s}$ & $\ldots$ & $\cdots$ & $3 s+1$ \\
\hline$\ldots$ & $\ldots$ & $\cdots$ & $\ldots$ \\
\hline$\left[\frac{(2 k+1) p-4}{2}\right] s+1$ & {$\left[\frac{(2 k+1) p-4}{2}\right] s+2$} & $\ldots$ & {$\left[\frac{(2 k+1) p-2}{2}\right] s$} \\
\hline$\left[\frac{(2 k+1) p}{2}\right] s$ & {$\left[\frac{(2 k+1) p}{2}\right] s-1$} & $\cdots$ & {$\left[\frac{(2 k+1) p-2}{2}\right] s+1$} \\
\hline
\end{tabular}

From the above table

$\sum_{e \in E\left(F_{1}\right)} l_{1}(e)=1+2 s+2 s+1+4 s+\cdots+\frac{(2 k+1) p s}{2}$

$$
\begin{aligned}
& =2[2 s+4 s+\cdots+(p-2) s]+ \\
& \quad\left[1+1+\cdots+\frac{(2 k+1) p}{4}\right]+\frac{(2 k+1) p s}{2} \\
& =4 s\left[1+2+\cdots+\frac{(2 k+1) p-4}{4}\right]+ \\
& \quad \frac{(2 k+1) p}{4}+\frac{(2 k+1) p s}{2} \\
& =\frac{s}{8}[(2 k+1) p]^{2}+\frac{(2 k+1) p}{4} \\
& =\frac{(2 k+1) p}{8}[(2 k+1) p s+2]
\end{aligned}
$$

Similarly, we can calculate $\sum l_{i}(e)$ for each factor $F_{\mathrm{i}}$, $i=2,3,4, \cdots s$ in the decomposition, which is a constant i.e., $k_{e}=\frac{(2 k+1) p}{8}[(2 k+1) p s+2]$ for all $i=1,2,3, \cdots s$.

By Lemma 1.4, this labeling gives a $(2 k+1)$-factor-Esuper magic labeling and thus every $2(2 k+1) r$-regular graph of $2[(2 k+1) r+1]$ order is $(2 k+1)$-factor-Esuper magic decomposable.

\subsubsection{Example}

A 6-regular graph $\mathrm{G}$ of order 8 is 3 -factor-E-super magic decomposable.

Here $k=1$

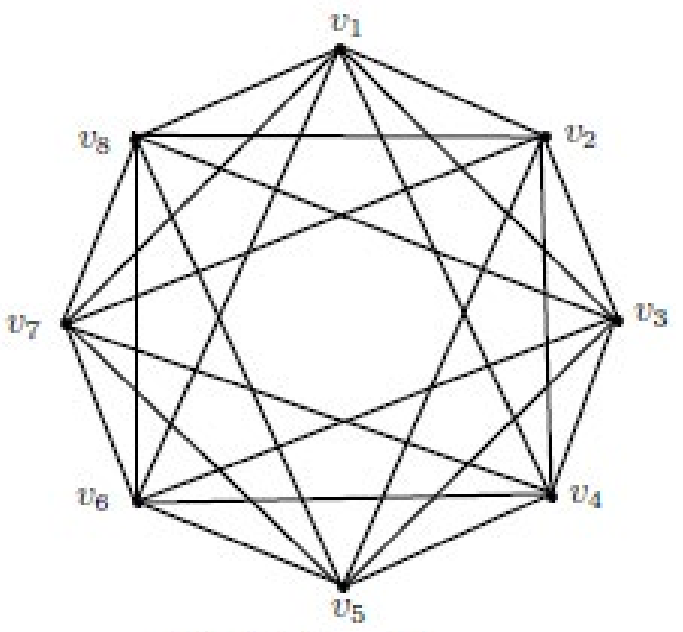

Fig 1.1(a) $G$ 
The graph $\mathrm{G}$ is decomposed into two 3-factors i.e., $F_{1}$ and $F_{2}$.

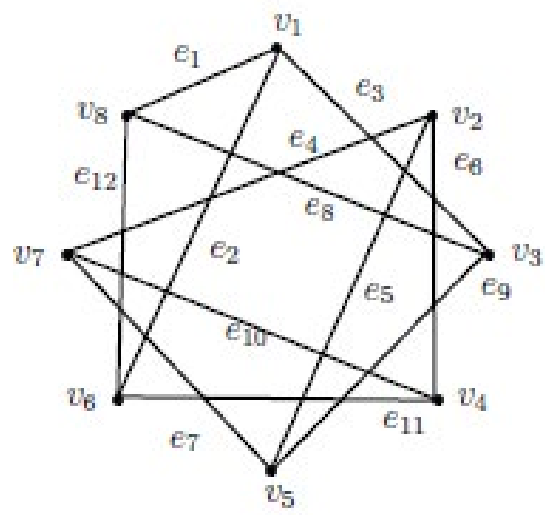

$F_{1}$

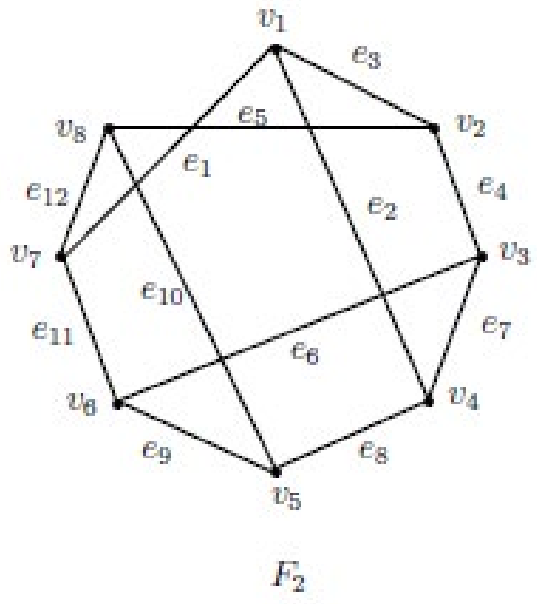

Fig $1.1(\mathrm{~b})$

Now $\sum l_{i}(e)=k_{e}=150$ for $i=1,2$

\begin{tabular}{|l|l|l|l|l|l|l|}
\hline & $e_{1}$ & $e_{2}$ & $e_{3}$ & $e_{4}$ & $e_{5}$ & $e_{6}$ \\
\hline$l_{1}(e)$ & 1 & 4 & 5 & 8 & 9 & 12 \\
\hline$l_{2}(e)$ & 2 & 3 & 6 & 7 & 10 & 11 \\
\hline
\end{tabular}

\begin{tabular}{|l|l|l|l|l|l|l|}
\hline$e_{7}$ & $e_{8}$ & $e_{9}$ & $e_{0}$ & $e_{11}$ & $e_{12}$ & $k_{e}=\sum_{e \in F} l_{i}(e)$ \\
\hline 13 & 16 & 17 & 20 & 21 & 24 & 150 \\
\hline 14 & 15 & 18 & 19 & 22 & 23 & 150 \\
\hline
\end{tabular}

Thus, from Lemma 1.4, G is 3-factor-E-super magic decomposable.

\subsection{Theorem}

Every graph $\mathrm{G}$ is $(2 k+1)$-factor E-super magic decomposable if and only if $\mathrm{G}$ is $3(2 k+1) r$-regular with order $3(2 k+1) r+1$ for $k \geq 1$ and $r=2 k-1$.

\subsubsection{Proof}

Necessity follows from Theorem 2.1. Let $G$ be an $3(2 k+1) r$-regular graph of order $p=3(2 k+1) r+1$ . i.e., $\mathrm{G}$ is an odd regular graph with even order. Then by Theorem 1.5, G is decomposed into $(2 k+1)$-factors $F_{1}, F_{2}, F_{3} \cdots F_{s}$, where $F_{i}$ is a $(2 k+1)$ factor for each $i, 1 \leq i \leq s$. Since $\mathrm{G}$ is of order $p$ with $s$ number of $(2 k+1)$-factors, the total number of edges of $\mathrm{G}$,

$q=\frac{(2 k+1) p s}{2}$.

If $l_{i}(e)$ denotes the labels of the edge of the factor $F_{i}$ in the decomposition. Now the labeling of edges in $G$ is as follows:

\begin{tabular}{|l|l|l|l|}
\hline$l_{1}(e)$ & $l_{2}(e)$ & $\cdots$ & $l_{s}(e)$ \\
\hline$s^{2}+1$ & $s^{2}+2$ & $\cdots$ & $s^{2}+s$ \\
\hline$s^{2}+2 s$ & $s^{2}+2 s-1$ & $\cdots$ & $s^{2}+s+1$ \\
\hline$s^{2}+2 s+1$ & $s^{2}+2 s+2$ & $\cdots$ & $s^{2}+3 s$ \\
\hline$s^{2}+4 s$ & $s^{2}+4 s-1$ & $\cdots$ & $s^{2}+3 s+1$ \\
\hline$\cdots$ & $\cdots$ & $\cdots$ & $\cdots$ \\
\hline$\left[\frac{(2 k+1) p-4}{2}\right] s+1$ & {$\left[\frac{(2 k+1) p-4}{2}\right] s+2$} & $\cdots$ & {$\left[\frac{(2 k+1) p-2}{2}\right] s$} \\
\hline$\left[\frac{(2 k+1) p}{2}\right] s$ & {$\left[\frac{(2 k+1) p}{2}\right] s-1$} & $\cdots$ & {$\left[\frac{(2 k+1) p-2}{2}\right] s+1$} \\
\hline
\end{tabular}

From the above table $\sum l_{1}(e)$,

$$
\begin{array}{r}
\sum_{e \in E\left(F_{1}\right)} l_{1}(e)=M N+s^{2}+1+s^{2}+2 s+s^{2}+2 s+1+ \\
s^{2}+4 s+\cdots+\left[\frac{(2 k+1) p-2 s-4}{2}\right] s+1
\end{array}
$$




$$
+s^{2}+\left[\frac{(2 k+1) p-2 s}{2}\right] s
$$

where $M N$ is magic number of $s \times s$ magic squares

$$
\begin{aligned}
&= M N+\left[\frac{(2 k+1) p-2 s}{2}\right] s^{2}+ \\
& 2\left(2 s+4 s+\cdots+\left[\frac{(2 k+1) p-2 s-4}{2}\right] s\right]+(1+1+\cdots 1)+\left[\frac{(2 k+1) p-2 s}{2}\right] s \\
&=\frac{s^{3}}{2}+\frac{s}{2}+\frac{(2 k+1) p s^{2}}{2}-s^{3}+\frac{((2 k+1) p)^{2} s}{8} \\
& \quad-\frac{(2 k+1) p s^{2}}{2}+\frac{s^{3}}{2}-\frac{(2 k+1) p s}{2}+s^{2} \\
& \quad+\frac{(2 k+1) p}{4}-\frac{s}{2}+\frac{(2 k+1) p s}{2}-s^{2} \\
&=\frac{(2 k+1) p}{8}[(2 k+1) p s+2]
\end{aligned}
$$

Similarly, we can calculate $\sum l_{i}(e)$ for each factor $F_{i}$, $i=2,3, \cdots s$ in the decomposition is the constant i.e.,

$k_{e}=\frac{(2 k+1) p}{8}[(2 k+1) p s+2]$.

By Lemma 1.4, this labeling gives a $(2 k+1)$-factor-Esuper magic labeling of $\mathrm{G}$.

\subsubsection{Example}

A 9- regular graph $\mathrm{G}$ of order 10 is 3-factor-E-super magic decomposable. Here $k=1$ and $p=10$

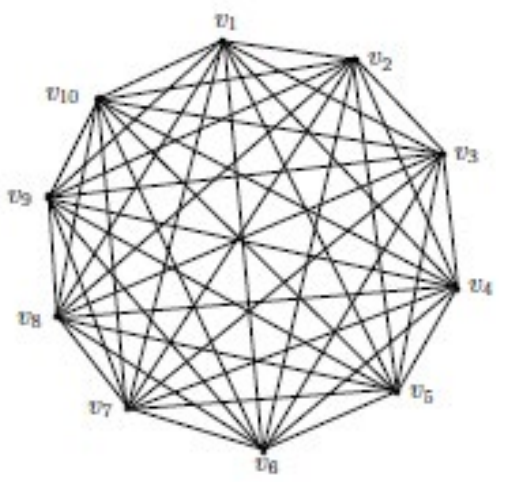

Fig 1.2(a) G

The graph $\mathrm{G}$ is decomposed into three 3-factors. $F_{1} F_{2}$ and $F_{3}$.
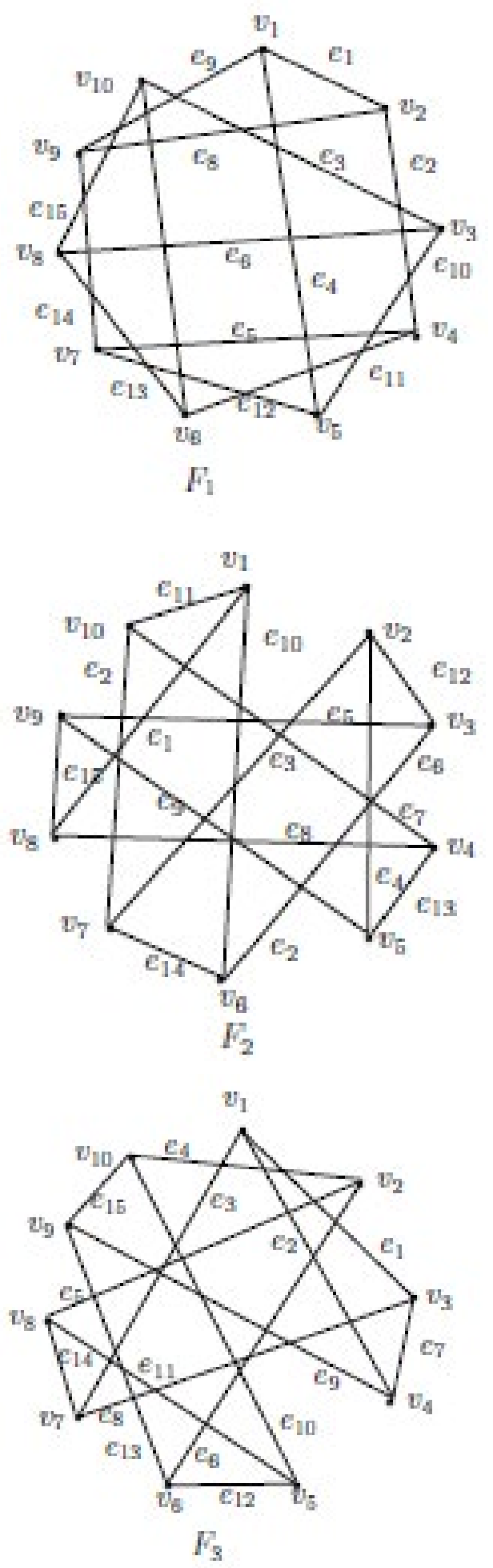

Fig $1.2(\mathrm{~b})$

Now $\sum l_{i}(e)=k_{e}=345$ for each factor $F_{i}, i=1,2,3$.

Next theorem shows that if $\mathrm{G}$ is a regular graph of odd order then $\mathrm{G}$ is not $(2 k+1)$-E-super magic decomposable for $k \geq 1$. 


\begin{tabular}{|l|l|l|l|l|l|l|l|l|l|l|l|l|l|l|l|l|}
\hline & $e_{1}$ & $e_{2}$ & $e_{3}$ & $e_{4}$ & $e_{5}$ & $e_{6}$ & $e_{7}$ & $e_{8}$ & $e_{9}$ & $e_{\mathrm{0}}$ & $e_{\mathrm{1}}$ & $e_{\mathrm{D}}$ & $e_{\mathrm{B}}$ & $e_{\mathrm{\sharp}}$ & $e_{\mathrm{5}}$ & $k_{e}=\sum_{e \in F} l_{i}(e)$ \\
\hline$l_{1}(e)$ & 4 & 9 & 2 & 10 & 15 & 16 & 21 & 22 & 27 & 28 & 33 & 34 & 39 & 40 & 45 & 345 \\
\hline$l_{2}(e)$ & 3 & 5 & 7 & 11 & 14 & 17 & 20 & 23 & 26 & 29 & 32 & 35 & 38 & 41 & 44 & 345 \\
\hline$l_{3}(e)$ & 8 & 1 & 6 & 12 & 13 & 18 & 19 & 24 & 25 & 30 & 31 & 36 & 37 & 42 & 43 & 345 \\
\hline
\end{tabular}

\subsection{Theorem}

If $\mathrm{G}$ is an $2(2 k+1) r$ and $3(2 k+1) r$-regular graph of odd order then $\mathrm{G}$ is not $(2 k+1)$-factor-E-super magic decomposable, for $k \geq 1$.

\subsubsection{Proof}

Let $\mathrm{G}$ be an $2(2 k+1) r$ and $3(2 k+1) r$-regular graph of odd order $p$ and size $q$ Suppose $\mathrm{G}$ is an $(2 k+1)$ -factor-E-super magic decomposable graph. Then $\mathrm{G}$ has an $(2 k+1)$-factor-E-super magic labeling and hence $k_{e}=\frac{q(q+1)}{2 s}$, where $s$ be the number of $m$-factors by Lemma 1.3,. Since G is $(2 k+1)$-factor decomposable with $q=\frac{(2 k+1) p s}{2}$. We have,

$$
\begin{aligned}
& k_{e}=\frac{(2 k+1) p s}{2}\left[\frac{(2 k+1) p s}{2}+1\right] \frac{1}{2 s} \\
= & \frac{1}{8 s}\left[((2 k+1) p s)^{2}+2(2 k+1) p s\right] \\
= & \frac{(2 k+1)^{2} p^{2} s}{8}+\frac{2(2 k+1) p}{8}
\end{aligned}
$$

which is an integer only if $p$ is even. Otherwise, if $p=2 t+1$, an odd integer then $k_{e}$ is not an integer. Hence by Lemma 1.4, G does not have an $(2 k+1)$-factor-E-super magic decomposition if $p$ is odd.

\section{Conclusion}

In this paper, a necessary and some sufficient conditions are obtained for some even regular graphs $\mathrm{G}$ of even order and for some odd regular graphs $\mathrm{G}$ of even order to have an $(2 k+1)$-factor-E-super magic decomposition for $k \geq 1$. In future, the problem of F-E or F-V anti-magic decomposition of graphs can be carried out.

\section{References}

1. Andrews W S. Magic Squares and Cubes. Dover. 1960.

2. Marr A M. and Wallis W D. Magic Graphs. Second Edition. Boston: Birkhauser-Basel; 2013. https://doi.org/10.1007/9780-8176-8391-7

3. Gallian J A. A Dynamic Survey of Graph Labeling. The Electronic Journal of Combinatorics. 2009; 16: DS6

4. MacDougall J A, Miller M, Slamin, Wallis W D. Vertex Magic Total Labeling of Graphs. Util.Math. 2002; 61: 3-21.

5. Marimuthu G, Balakrishnan M. E-Super Vertex Magic Labeling Of Graphs. Discrete Appl. Math. 2012; 160: $1766-$ 74. https://doi.org/10.1016/j.dam.2012.03.016

6. Petersen J. Die Theories Der Regularen Graphs. Acta Math. 1891; 15: 193-220. https://doi.org/10.1007/BF02392606

7. Sedlacek J. On Magic Graphs. Mathematica Slovaca. 1976; 26: 329-335.

8. Subbiah S P, Pandimadevi J. H-E Super Magic Decomposition of Graphs. Electronic Journal of Graph Theory and Applications. 2014; 2: 115-128. https://doi.org/10.5614/ ejgta.2014.2.2.4

9. Swaminathan V, Jeyanthi P. Super Vertex-Magic Labeling. Indian J. Pure and App. Math. 2003; 34(6): 935-939.

10. Wang T M, Zhang G H. Note on E-Super Vertex Magic Graphs. Discrete Applied Mathematics. 2014; 178: 160-2. https://doi.org/10.1016/j.dam.2014.06.009 